\title{
BMJ Open Living with COVID-19: a phenomenological study of hospitalised patients involved in family cluster transmission
}

\author{
Wei Liu (D) , ${ }^{1}$ Jia Liu ${ }^{2}$
}

To cite: Liu W, Liu J. Living with COVID-19: a phenomenological study of hospitalised patients involved in family cluster transmission. BMJ Open 2021;11:e046128. doi:10.1136/ bmjopen-2020-046128

- Prepublication history for this paper is available online. To view these files, please visit the journal online (http://dx.doi org/10.1136/bmjopen-2020046128).

WL and JL contributed equally.

WL and JL are joint first authors.

Received 21 October 2020

Revised 22 January 2021

Accepted 08 February 2021

A) Check for updates

(C) Author(s) (or their employer(s)) 2021. Re-use permitted under CC BY-NC. No commercial re-use. See rights and permissions. Published by BMJ.

${ }^{1}$ Adelphi University College of Nursing and Public Health, Garden City, New York, USA

${ }^{2}$ The Third Xiangya Hospital, Central South University, Changsha, Hunan, China

\section{Correspondence to} DrWei Liu; wliu@adelphi.edu and

Ms. Jia Liu;

jialiu1980@csu.edu.cn

\section{ABSTRACT}

Objectives To describe experiences of hospitalised patients with COVID-19 following family cluster transmission of the infection and the meaning of these experiences for them.

Design A descriptive phenomenological design was used to construct themes depicting patients' experiences of living with COVID-19.

Setting This study was conducted in a major teaching hospital in Wuhan, China, in March 2020.

Participants Fourteen patients involved in family cluster transmission of COVID-19 were recruited into the study. The participants consisted of seven males and seven females. Data were collected through semistructured, indepth, face-to-face interviews. Interviews were transcribed verbatim and analysed using Colaizzi's approach. Results Six themes emerged from data analysis during two distinct phases of patients going through COVID-19: the early outbreak phase and the later hospitalisation phase. Early in the outbreak, patients experienced life imbalances between individual well-being and family responsibilities. While facing widespread prejudice and rejection, patients dealt with the heavy toll that the illness had left on their body and mind. After being hospitalised, patients described feelings of living with uncertainty, sadness, fear of death and concerns about family, while simultaneously hoping for a better life after recovery. Conclusions Our findings suggest that living with COVID-19 is an emotionally and physically challenging experience for patient participants in the study. Psychological evaluations need to be routinely carried out with patients in a public health crisis. Interprofessional and interorganisational collaborative efforts should be made to examine the physical and psychological sequelae of COVID-19, as well as investigate outcomes of existing intervention programmes.

\section{INTRODUCTION}

In December 2019, a novel coronavirus disease later known as COVID-19 emerged in Wuhan, the capital city of Hubei province in China. Within 1 month, the deadly viral disease spread from a single city to the entire country. ${ }^{1}$ Because of its strong human-tohuman transmission ability, COVID-19 soon became a global public health concern. ${ }^{2}$ On
Strengths and limitations of this study

- Relatively few studies have been conducted to explore patient experiences of living with COVID-19.

- Working with patients at the bedside enabled us to identify the research needs of our patients and motivated us to undertake the inquiry into this vulnerable population.

- The major strength of this study is the phenomenological approach used to describe hospitalised patients' thoughts, health situations, feelings and behaviours amidst the infectious outbreak.

- Our sample population consisted of patients with mild to moderate clinical symptoms, which limits the transferability of our findings to the COVID-19 patient population at large.

11 March 2020, the WHO officially declared COVID-19 a global pandemic. ${ }^{3}$ In the meantime, global collaborative research efforts have been made to share real-time data combating the pandemic. The pertinent research on the viral disease concerning its clinical and epidemiological features and possible transmission routes is ever-expanding. ${ }^{45}$ Even the lived experiences of frontline healthcare workers caring for patients with COVID-19 are growing rapidly. ${ }^{67}$ However, relatively few studies have been conducted to explore the real-life experiences of those affected by the illness, especially when they are admitted to the hospital.

The earliest report on patients' experiences of living with COVID-19 was documented by Sahoo $e t a l^{8}$ in October 2020. The case descriptions of three patients revealed highly distressing mental health issues that patients experienced on hospital isolation units. Within the available literature on patients' lived experiences, Shaban and colleagues ${ }^{9}$ hermeneutical inquiry of 11 patients with COVID-19 provides a rich insight into how patients dealt with their emotions in the 
context of hospital isolation. The researchers suggested changes to physical environments and development of care models to minimise the negative consequences of isolation in healthcare settings. Most recently, the body of work has shifted from patients to survivors primarily focusing on their health-related beliefs, ${ }^{10}$ along with their persistent physical and psychological issues during and after COVID-19. ${ }^{11}{ }^{12}$ In light of the resurgence of the pandemic in many countries around the world, there is an increased need to glean more insight into the lifeworld of those vulnerable patients in order to inform evidencebased clinical practice. ${ }^{13}$

Early in the outbreak of the pandemic, many case studies reported in China have demonstrated consistent evidence on rapid transmission of COVID-19 in the form of family clusters. ${ }^{45}$ Consequently, extraordinary public health measures and education campaigns have been implemented to reduce further spread of the virus within China and elsewhere. ${ }^{2}$ While there have been studies on the biomedical aspects of the illness and the experiences of healthcare workers and survivors, relatively few studies have been done to explore patient experiences in the midst of COVID-19. A better understanding of patients' lived experiences might help to inform local and national efforts to strengthen service provision for patients and their families during and after the outbreak. Healthcare workers will also benefit from the perspectives of the recipients of their care in preparation for the current resurgence of COVID-19 and future pandemics. ${ }^{14}$ Given that COVID-19 is transmitted quickly within family clusters, we endeavoured to explore the experiences of patients originated from family cluster contexts to clarify the individual and family impact of the outbreak. The central research question guiding the study was: 'What is the experience of living with COVID-19 following family cluster transmission of the illness?'

\section{METHODS}

\section{Design}

Descriptive phenomenology was the research design selected for the study. Husserl's philosophical underpinnings are foundational to descriptive phenomenology. ${ }^{15}$ Husserl's approach has been adopted to shed new light on nurses' experiential accounts in caring for patients with COVID-19. ${ }^{616}$ For Husserl, the aim of phenomenology is to arrive at an essential understanding of human consciousness through describing features common to all people who have the experience. ${ }^{17}$ Descriptive phenomenology is most suitable to make an inquiry into universal aspects of a phenomenon that have never been conceptualised in prior research.$^{18}$ Living with COVID-19 is a new phenomenon, and existing literature on the outbreak does not readily inform us about the inherent complexities and intricacies of patients' emotional responses to this unprecedented crisis.

Inherent in descriptive phenomenology is the principle of bracketing by holding in abeyance preconceived beliefs and opinions about the phenomenon under study. ${ }^{15}$ Through bracketing, the essence of the phenomenon emerges from the perspective of those experiencing it. Bracketing for this study was accomplished through reflexive journalling. Throughout the study, reflexive notes regarding preconceptions and presuppositions, experiences of the research team caring for patients with COVID-19, together with thoughts after interviews were recorded and discussed among members of the research team during our daily WeChat video conferences. Because of the dual role of the members of the research team as nurses and researchers, we intended to meet the phenomenon as rigorous and unbiased as possible. Descriptive phenomenology offered us the methodological tools to refrain from our judgement and focus on describing the lived experience as opposed to interpreting it. Furthermore, reflexive notes were revisited prior to each interview and during data analysis to ensure the interviewer-imposed assumptions did not take precedent over the participants' described experiences.

\section{Setting and participants}

The study was conducted in a large teaching hospital in Wuhan, China. Our research team primarily consisted of nurses who were deployed to Wuhan from another teaching hospital in Hunan province to work intensively with this patient population. Following our deployment to Wuhan, members of the research team were assigned to work in a 55-bed occupancy general isolation ward where patients were admitted with mild to moderate COVID-19 symptoms for isolation or transferred from intensive care units (ICUs) waiting for discharge. All patients on the general isolation ward were able to perform activities of daily living independently or with minimal assistance. The patients were free to move around the public areas on the isolation ward such as hallways and tea rooms while wearing face masks and keeping physical distance. While working with patients closely on the isolation ward, members of the research team were constantly intrigued by the patients' needs to share their stories with frontline healthcare workers, particularly nurses. Initially, we were concerned about our ability to meet the phenomenon completely unprejudiced. However, the merits of conducting this research outweighed any potential harm to scientific rigour. Finally, we decided to take this unique opportunity and apply rigorous research methods to investigate the lived experiences of those patients that we cared for.

To concentrate on our clinical responsibilities and meet the phenomenon as free as possible, we assigned specific nursing staff members to recruit participants and conduct interviews. The sample criteria required that the patient was involved in family cluster transmission; was willing and able to articulate his or her experience; and had stable physical conditions (eg, oxygenation index $\geq 300 \mathrm{~mm} \mathrm{Hg}$ ). In addition, healthcare providers' opinions were sought to determine the appropriateness of patients' participation in the study. Participants were 
recruited using a combination of convenience, snowball and purposive sampling strategies. Our first three participants responded to the research flyers posted in the public areas of the general isolation ward. The rest of the participants were recruited through snowball sampling. To maximise the sample variations, we purposively took into consideration of the index case in the family, the number of patients in each family cluster and the number of deaths in the family. In addition, we purposively recruited all participants from different family clusters attempting to generate rich perspectives because of the exploratory nature of the study. Patients' experiences from the same family clusters are distinctive ones with an additional layer of complexities and dynamics, warranting a separate study of this potentially vast population.

\section{Ethics}

All participants were informed of the voluntary nature of the study, and they were allowed to withdraw from the study at any time without any consequences to the care provided to them. Because of the sensitive nature of the research topic, participants might be referred to a mental health counsellor in the hospital if they showed strong emotional responses to the interview questions. The referral would only be made with individual participant's permission. Furthermore, the interviewer showed participants' empathy throughout the interviews and constantly bracketed out her personal feelings. Written consent was obtained from all participants before the start of the interview. Confidentiality was ensured by assigning a unique participant number to replace participants' names on the transcripts. All electronic data were kept in passwordprotected files on computers, which were in a secure place and only accessed by members of the research team. The raw data (eg, reflexive notes and audio-recordings) were stored separately in a locked facility.

\section{Data collection}

Semistructured, in-depth, face-to-face interviews were conducted between 1 March and 25 March 2020. All interviews were conducted in a private, quiet conference room on the isolation ward to ensure privacy and participant comfort and to promote open sharing of experiences. During the entire interview, both the interviewer and the interviewee wore new face masks and sat apart from each other with 6 feet physical distance. Each interview lasted between 40 and $60 \mathrm{~min}$, and it was audio-recorded. Demographic information about each participant was obtained at the start of the interview. Interviews began with a broad open-ended question asking, 'What is it like to be a patient living with COVID-19?'. Prompts from the interview guide were used to encourage participants to elaborate on their experiences (box 1). Having daily interactions with patients on the unit helped the research team to develop interview questions that were most pertinent to patients' experiences living with COVID-19.

To separate our researcher and caregiver roles, we recruited a master's degree prepared nursing staff

\section{Box 1 Interview guide}

1. Can you tell me about your overall experiences after being infected?

2. How has your family role changed after being infected?

3. Reflecting on your personal experience, how has this changed your family responsibilities and/or relationships, and what has been the biggest challenge for you and your family?

4. Going forward, how do you think this experience would impact future life for you and your family?

member who was not directly affiliated with the study to conduct all interviews. Prior to the conduct of patient interviews, the nursing staff organised practice interviews with a qualitative researcher on the research team to validate appropriate interview skills, and bracketing was implemented throughout the investigation. The audiorecording was transcribed verbatim by two members of the research team within 24 hours, and the interviewer's reflexive notes were discussed among members of the research team. The interviews and original transcripts were originally recorded in Chinese. All quotations were translated into English language by the first author (female) and back translated into Chinese language by the co-first author (female) to assess semantic equivalence between the original and back translated versions of the transcripts. ${ }^{19}$ Data collection continued until data saturation had been achieved as evidenced by redundant information kept emerging.

\section{Data analysis}

All audio and written data were imported into NVivo V.12 (QRS International) to assist with data management and categorisation. The analysis based on Colaizzi's method included the following seven steps: (1) reading all the transcripts three to five times to gain an understanding of meanings conveyed; (2) reviewing each description and extracting significant statements; (3) formulating meanings for these significant statements; (4) categorising the formulated meanings into clusters of themes; (5) integrating the findings into an exhaustive description of the phenomenon; (6) returning the exhaustive description to participants for validation of feelings; and (7) incorporating any relevant new data into final description of the essence of the phenomenon. ${ }^{20}$ In the present study, 150 significant statements were extracted from the transcribed interviews. These significant statements were then transformed into 42 formulated meaning units. These formulated meaning units were later grouped into two main categories with six distinct themes discussed in the findings. Initial data coding was completed by two members of the research team. Nevertheless, there were ongoing discussions among all research team members about the analysis of the data, and the research team reached an agreement on final findings presented in this paper. Throughout the paper, we followed the Consolidated Criteria for Reporting Qualitative Research. ${ }^{21}$ 


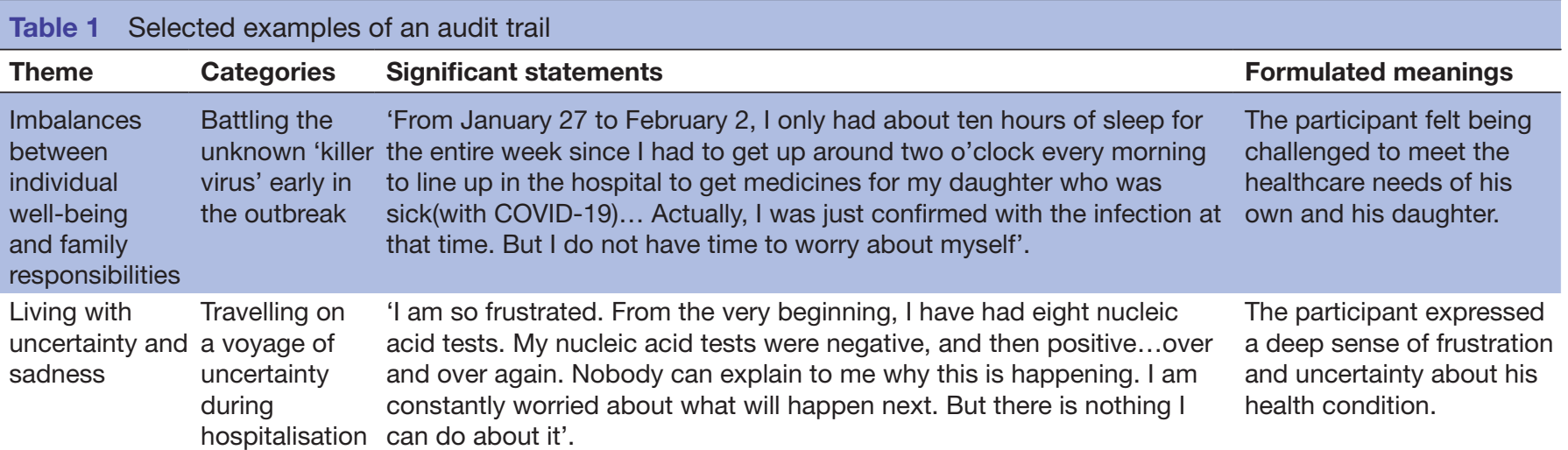

\section{Trustworthiness}

To enhance trustworthiness of the study, we followed Lincoln and Guba's definitions for the establishment of rigour in qualitative research. ${ }^{22}$ To ensure credibility, bracketing was implemented throughout the research process to put aside prior personal knowledge and experience about the phenomenon. In addition, adequate time was spent with each participant, which was followed by intense engagement with the raw data. To support transferability, we provided rich and thorough descriptions of participants' experiences citing their verbatim statements. Dependability was ensured by a clear audit trail demonstrating how analytic decisions were made (table 1). Confirmability was established by sharing the essential categories and themes with each participant. By the time all the interviews were completed, some of the participants had been discharged from the hospital. We conducted member checking by telephoning all the participants instead of returning our exhaustive description of the phenomenon to them. Although this was a slight modification of Colaizzi's method, all participants agreed that the identified themes captured the essence of their experiences of being a patient with COVID-19 involved in family cluster transmission of the illness.

\section{Patient and public involvement}

Patients were involved in the design, conduct and analysis phases of the research. Working with patients at the bedside enabled us to identify the research needs of this patient population and motivated us to undertake this research endeavour. While developing the interview guide, the research team received input from patients to ensure the appropriateness and relevance of the interview questions. As initial themes emerged, we reviewed the results with all patient participants to verify the findings and seek their additional feedback.

\section{RESULTS}

Fourteen participants were recruited into the study. The sample consisted of seven males and seven females across an age range of 30-73 years. In the sample, four participants were the index case in the family, while the other 10 participants contracted the disease from a close family member. The participants reported that two to five family members had been diagnosed with COVID-19 at the time of the study, with death toll reported in some participants' families (table 2). Patients with COVID-19 involved in family cluster transmission in the study revealed emotional experiences that occurred during two distinct phases of their journey battling the infectious disease: the early outbreak phase and the later hospitalisation phase. During each phase, three themes captured the essence of the participants' lived experiences.

\section{Battling the unknown 'killer virus' early in the outbreak}

In the early outbreak, participants described a variety of emotions combating this fatal new virus, characterised by feelings of stress, hopelessness, vulnerability, rejection and guilt. The following three themes and the accompanying verbatim expressions illuminated participants' feelings of emotions.

\section{Imbalances between individual well-being and family responsibilities}

Following the outbreak in Wuhan in January 2020, participants described that COVID-19 had expunged their normal existence. All participants in the study experienced significant imbalances of two basic life components between individual well-being and family responsibilities. Some participants went extra miles and made huge sacrifices to take care of their infected family members at the expense of neglecting their own illness. The sense of imbalance was captured in one participant's quotes:

From January 27 to February 2, I only had about ten hours of sleep for the entire week since I had to get up around two o'clock every morning to line up in the hospital to get medicines for my daughter who was sick [with COVID-19]... Actually, I was just confirmed with the infection at that time. But I do not have time to worry about myself. (Participant 2)

Early in the COVID-19 outbreak, because of the extremely scarce healthcare resources available in Wuhan, many asymptomatic patients stayed at home under self-isolation, which significantly increased the risk of person-to-person transmission of the infection within family clusters. To protect their family members, some 
Table 2 Demographics of the participants

\begin{tabular}{llllll}
\hline & Gender & Age (years) & Index case* & $\begin{array}{l}\text { Number of patients } \\
\text { in the family cluster }\end{array}$ & $\begin{array}{l}\text { Death in the family cluster } \\
\text { from CoVID-19 }\end{array}$ \\
\hline Participant 1 & Female & $70-75$ & $\begin{array}{l}\text { The eldest } \\
\text { daughter }\end{array}$ & $\begin{array}{l}\text { The eldest daughter and } \\
\text { husband }\end{array}$ & Mother and father \\
\hline Participant 2 & Male & $45-50$ & Father & 4 & No one \\
\hline Participant 3 & Female & $50-55$ & Husband & 2 & No one \\
\hline Participant 4 & Female & $50-55$ & Patient self & 3 & No one \\
\hline Participant 5 & Male & $70-75$ & Patient self & 2 & Husband \\
\hline Participant 6 & Female & $55-60$ & Husband & 2 & No one \\
\hline Participant 7 & Female & $50-55$ & Patient self & 5 & No one \\
\hline Participant 8 & Male & $30-35$ & Father-in-law & 2 & No one \\
\hline Participant 9 & Male & $65-70$ & Patient self & 2 & Wife \\
\hline Participant 10 & Male & $70-75$ & Wife & 2 & Mother-in-law \\
\hline Participant 11 & Female & $40-45$ & Mother-in-law & 3 & Wife \\
\hline Participant 12 & Male & $60-65$ & Wife & 2 & Father \\
\hline Participant 13 & Female & $35-40$ & Father & 3 & Mother \\
\hline Participant 14 & Male & $55-60$ & Mother & 4 & \\
\hline
\end{tabular}

*Index case was the first case tested positive in the family cluster.

participants desperately searched for a bed in a hospital so that they would not infect their loved ones at home. While reminiscing over his experience, one patient recalled:

There were patients everywhere, but there were no [hospital] beds. I looked around all the hospitals in Wuhan and called so many friends, and I finally got a [hospital] bed... I have a four year old in my family. I am afraid of giving him the virus. So I ran away to protect my family. I haven't been able to see them for almost two months. (Participant 5)

\section{Facing widespread prejudice and rejection}

Having an unknown infectious disease made the participants feel being discriminated against by the public and even healthcare workers in the early stage of the outbreak. Many participants experienced rejection by fearful healthcare workers while seeking medical help. The sense of belittlement was best illustrated in the words of one participant:

When we went to the hospital the first time in January, they [healthcare workers] really treated us [the patient and her husband] like bad guys, like mice, rotten mice. It felt like you [the patient and her husband] were so dirty, and you should not be near me. I cannot really tell you [the interviewer] the feeling. (Participant 3)

People's desire to avoid infection and remain healthy resulted in obvious behaviours of social rejection. Having multiple family members infected added another layer of complexities to the social pain and distress, as described by the following participants:

Three of us in the family were infected by the virus, and my mother-in-law died. People were just scared of us... My whole family have not contacted our relatives and friends out of Wuhan for more than one month. Everyone calls it 'Wuhan Virus.' We are 'Wuhan Virus' that can kill others. The prejudice is painful. (Participant 11)

My daughter was infected after coming back to Wuhan for the Chinese Spring Festival. She said she would never come back to Wuhan again after her grandparents died [because of COVID-19]... She said there are too many painful memories in Wuhan. Everything about the family and the city [Wuhan] had changed to her. (Participant 2)

Heavy toll on the body and mind

COVID-19 took a heavy toll on the participants' body and mind. All the participants experienced unforgettable physical and psychological trauma in the early phase of the outbreak. The psychological impact came through clearly in the quotes of one patient as she described a deep sense of guilt for her father's death.

On December 28, 2019, my family went to a friend child's tenth birthday party. At the party, there was another old couple who were relatives of our family. The couple died within 20 days after the gathering because of the virus... I was so scared when my father was tested positive. That was like a 'death sentence' because there was no hope to get him a hospital bed 
then. His condition deteriorated in a few days, and he died at home. I feel a lot of agony and pain in my heart. I cannot escape from the guilt. It feels like a monster living inside of me. (Participant 13)

Other patients described a high level of fear and anxiety for their own physical health, as recalled by one participant:

When I went to the hospital that day, I coughed and had a lot of phlegm. I waited for hours to see a doctor. Finally, I had a checkup, CT [computerized tomography] scan, and the nurse took my blood. I did not get out of the hospital until 11 o'clock at night. There were no public transportation or Taxi services. Everything was shut down. I had to walk home, or maybe crawl back [home], or die on my way home as I was trembling and shivering. (Participant 5)

While coping with the physical and psychological pain resulted from a deadly infectious disease, some participants experienced family loss and grief to an overwhelming degree since the early outbreak.

Both of my parents died in January from the virus. I was not myself, thinking I might just go with them. I felt empty inside... My daughter became sick a week later, and she got the infection from me. I did not want to die without knowing what would happen to her. (Participant 2)

\section{Travelling on a voyage of uncertainty during hospitalisation}

After being hospitalised, participants had more time to think about their own health issues and reflect on their experiences. The feelings of uncertainty, sadness, fear of death, concerns about family and hope for life prevailed in participants' description of their experiences. Three themes were dominant across participants' expression of emotions.

\section{Living with uncertainty and sadness}

Participants' perception of uncertainty was related to the unknown and unpredictable nature of the illness. With the ever-increasing number of confirmed and suspected cases reported in the country, widespread social media coverage on the crisis and a lack of effective standard treatment for the disease, participants expressed concerns about the long-term sequelae of the infection, possibilities of reoccurrence and whether it would have any impact on their future family life. Some of the participants' quotes captured their emotions:

People always talk about this virus. There are lots of rumors flying around. They are saying the virus will stay in your body forever just like the hepatitis B virus, and you will get sick again...' (Participate 12)

I was originally planning to have a baby this year. I had a CT [computerized tomography] scan, and they [doctors] told me that the virus had done some damage to my body, and there might be some long-term effects on other parts of my body. I will have to postpone the plan to expand my family anytime soon. (Participant 8)

In viewing the heavy emotional toll on the general public after the SARS outbreak in China in 2003, the State Council of China opened up nationwide psychological counselling hotlines in early February 2020. While staying on an isolation ward, all patients were able to use electronic devices and applications such as smartphones and WeChat to access free psychological counselling services anytime of the day. Nevertheless, some participants still felt living in a dark world of uncertainty and sadness:

I am so frustrated. From the very beginning, I have had eight nucleic acid tests. My nucleic acid tests were negative, and then positive... over and over again. Nobody can explain to me why this is happening. I am constantly worried about what will happen next. But there is nothing I can do about it. (Participant 2)

The city [Wuhan] has been locked down for more than one month. There were too many bad things that had happened during this time. I lost my daughter and my husband last month. My friends died. My relatives died. The pain and sadness are beyond recognition... I heard about the counselling hotline. But I do not think I need that. I just have to live with the pain. (Participant 1)

Living with fear of death and concerns about family

The constant threat of death from the illness persisted in participants' daily life. Having an unknown infection made participants feel extremely vulnerable to death. In addition, many participants in the study experienced physical challenges fighting the symptoms of the infection and/or unwanted side effects from medical treatment after being hospitalised. A patient recalled that:

When I took Aluvia [Lopinavir/Ritonavir Tablets], I could not eat anything for three days because of nausea and vomiting. I have severe chronic enteritis... I knew that I would die if I did not eat. But I would also die if I did not take the medicine. It felt like I would die either way. (Participant 14)

Some of the participants were transferred to the general isolation ward from COVID-19 ICUs. While receiving intensive care, the participants were exposed to daily death of other patients on the unit. Those were harrowing experiences to the participants inducing fear and sadness, as lamented by one participant:

You don't understand the pain in my heart when I found out she [another ICU patient] died that night. She and I were admitted around the same time... I saw her body was taken away, thinking that the next time I would be the one they would be taking. I cried so badly for a few days. It was heart-wrenching. (Participant 6) 
While admitted in the hospital, patients were able to access smartphones and wireless internet to communicate with their families. However, all participants felt great concerns about family life outside the isolation ward. The mandatory quarantine policy in a designated isolation centre added another burden, as participants knew that their infected family members had been separated from the rest of the community. The participants' concerns were reflected in their accounts:

All five of us in the family had been tested positive. We are in different hospitals and isolation centers I know my family will be treated differently by other people. People in small cities used to envy people from Wuhan, but now Wuhan is notoriously famous all over the world. (Participant 7)

My husband died in January. My son is at home by himself. He is fearless. He thinks that the virus will not get him. He has always been on my mind. I spoke to him on the phone yesterday. He said everything is fine. I have a feeling that he is hiding something from me. I just want to save him and myself from this crisis. (Participant 6)

\section{Living with hope and gratitude}

Despite the frightening and traumatising experiences of living with a fatal virus infection, many participants exhibited enormous resilience towards the threat and fear of the illness. Some participants described that COVID-19 had promoted their personal growth and motivations for a better life in the future. For example:

I have been staying in the hospital for over 40 days, and I have a lot of time to think about the true meaning of life and existence. I live with hope as I want to survive this and spend the rest of my life with my family in good health. Nothing is more important than that. (Participant 14)

Another participant described how living with COVID-19 had improved her family relationships:

My husband and I were not too happy with each other before the outbreak. After I got sick, I told him that he should remarry someone else if I could not make it. My husband told me off, and he said "we started the family together. We will walk through this together. I am here for you, and you are there for me. We will be living happily after this'. (Participant 11)

In addition to the positive state of seeking survival and a better family life, many participants felt that they were well taken care of by the hospital staff. By early March 2020, the hospital where the study was conducted was primarily staffed by healthcare workers from across China. After going through the most acute phase of the infection, many participants felt a deep sense of security and gratitude for the medical treatment and care they had received. While being admitted to an isolation ward, some patients perceived nurses as their family members, as the nurses helped them to eat, drink and go to the bathroom. As one participant expressed:

It is too hard for the nurses. They help us with everything since we do not have families with us... It must be so hot that their glasses [goggles] are sweaty. I cannot see their faces, but I remember all their names written on the back of their [personal protective] suits. They are like my daughters. (Participant 3)

Living through the experiences of battling COVID19, some participants extended empathy toward other patients and the community. As one participant recalled:

It was very late that night when I was driven to the hospital [by ambulance]. They [the ambulance staff] told us they did not know who they should hand off us to, and they just left... There was another elderly man in the ambulance. We had to walk inside the hospital lobby by ourselves. He cannot really walk. So I walked slowly with him. If I had not helped him, he would have to crawl into the hospital by himself. (Participant 9)

Another participant overheard the possibility of using convalescent plasma as a treatment for the illness and expressed his hope to save other patients' lives by donating his plasma:

I was very fit before I contracted the virus. I heard that some places are collecting plasma donations. If they still need the plasma, I can donate mine. I think I should be a good candidate for the donation. I am a soldier, and it is my nature to serve the country and the people. (Participant 10)

\section{DISCUSSION}

Our findings suggest that patients' experiences and emotions evolved as they moved through different phases of the pandemic. Early in the outbreak, the transmission and mortality rates of COVID-19 were considerably high in Wuhan because of the unknown nature of the illness and the extremely strained local healthcare system. To protect their families, patient participants made great sacrifices by exposing themselves to risks for their family members or leaving families behind to prevent them from being infected. Such a high level of family obligation and altruism was based on the Chinese social merit of collectivism and doing what is best for the family at the expense of individual well-being. ${ }^{23}$ Similarly, while under hospital care, some participants in the study lent helpful hands to other patients who were strangers. This finding is in line with earlier study among African Ebola survivors who identified patients caring for each other as a source of resilience for self-preservation, highlighting the prevalence of humanity and strength in crisis across different cultures and communities. ${ }^{14}$ Our findings further support Ladds and colleagues, ${ }^{12}$ call to establish online peer support groups during and after COVID-19 
to share patients' experiences and knowledge about the illness.

Participants in our study conveyed both negative emotions of feeling rejected in the early stage of the outbreak and positive experiences of receiving quality care during hospitalisation. A possible explanation for the change of participants' feelings might be attributed to the government's quick response efforts to strengthen the medical care capacity in Wuhan, which resulted in appropriate care provision to most of the patients. Our finding supports Liu and colleagues, ${ }^{, 7}$ emphasis on protecting healthcare workers from overwork and burnout during pandemic situations. Of note, the available literature also shows consistent evidence that survivors of COVID-19 experienced rejection and stigmatisation after recovering from the illness. ${ }^{10} 11$ The stigma was perceived to arise from the public suspicion that survivors still had the virus in their bodies, a similar concern that was expressed by some of our participants. To facilitate easy reintegration of survivors of COVID-19 into the community, it is important to develop clear communication messages to the public that survivors are no longer contagious and, in effect, require the utmost acceptance and care by members of the community. ${ }^{24}$ In addition, healthcare workers could accompany survivors home after discharge to support their safe returning to the community. ${ }^{25}$ It is equally important to create a positive environment in the community where the COVID-19 disease and its effects can be discussed openly and effectively. ${ }^{11}$

Following the outbreak in Wuhan in January 2020, the number of patients admitted to the regional and provincial hospitals increased substantially. To alleviate the strain on the healthcare system and boost the manpower of the local hospitals, 25633 healthcare workers from across China were deployed to Hubei province joining the fight against the outbreak as of 14 February 2020. ${ }^{26}$ Furthermore, the government constructed new field hospitals and converted local stadiums to makeshift treatment centres, known as Fangcang Hospitals. By midFebruary 2020, any individuals with asymptomatic or mild infection were mandatorily isolated at Fangcang Hospitals to reduce the person-to-person transmission in family clusters. If patients' conditions worsened, they were transferred to designated COVID-19 hospitals for further treatment. The decisions about interhospital transfers were made by the local government to ensure better coordination of care provision. Many patients in the study experienced separation from other infected family members because of the stratification admission policy based on the severity of clinical symptoms, which perpetuated their anxiety, despair and fear during hospitalisation. In addition, witnessing the death of families, relatives and other patients had been reported as a harrowing experience by participants in the present study, affirming survivors' experience of feeling being trapped between life and death while living with COVID-19. ${ }^{11}$ Although timely provision of mental healthcare was given the highest priority by the Chinese government since the early outbreak, ${ }^{27}$ the psychological counselling services easily accessible to patients were not fully used owing to a lack of recognition of mental health issues by some of the participants. This finding highlights that patient education about the importance of mental healthcare cannot be overemphasised in a large-scale public health crisis like COVID-19. All these issues also suggest that psychological evaluations and brief supportive sessions should be routinely carried out virtually by mental health professionals to help those patients who are going through the infection and/or experiencing family loss and grief. ${ }^{8}$ In addition, the essential personnel such as nurses who have the most frequent contact with patients should become more familiar with signs and symptoms of mental health issues and initiate early interventions by referring the patients to mental health professionals as needed.

In addition to the psychological counselling services, patients were encouraged to communicate with their families and friends using smartphones and WeChat for emotional support. Even with readily available ways of communication, many patient participants expressed great concerns about family life outside the isolation ward. Such findings were not surprising because of the fatal nature of the illness. While smartphones and WeChat facilitated easy communication between patients and family members, these communication channels also escalated the participants' stress levels. Throughout the outbreak, COVID-19 has been repeatedly described as a 'killer virus' on WeChat. ${ }^{27}$ In addition, nobody could escape media coverage on the rapidly increasing number of new cases every day. Already reported in other studies, patients with COVID-19 criticised sensationalised social media platforms for creating COVID-19 'infodemic' and exacerbated the anxiety level of the general public. ${ }^{911}$ Building on these findings, we encourage regular and accurate news updates from the local government and hospital officials about the outbreak. Nurses or other healthcare workers could telephone family members several times a week to update them and reassure them that their loves ones are cared for. In the meantime, we support the continued use of smartphones by patients in isolation to communicate with their family and friends and to establish a sense of connectedness with the world outside.

The feeling of uncertainty is a major theme in the participants' description of the essence of their experiences during hospitalisation. The existing literature shows that uncertainty is a common theme for those living throughCOVID-19. ${ }^{10} 12$ In the phenomenon of living with COVID-19, uncertainties of having a highly contagious disease, fear of death and possible long-term consequences imposed a severe stressful mental state on all the participants. In order to reduce fear and uncertainty, public educational campaigns should be launched immediately after the outbreak focusing on the diagnosis, help-seeking behaviours and prognosis of the illness. In addition, interprofessional and interorganisational collaborative efforts should be made to examine the physical 
and psychological sequelae of COVID-19, as well as investigate outcomes of existing intervention programmes. ${ }^{28}$

The participants' negative experiences associated with emotional turmoil in the early phase of the outbreak contrasted with their positive experiences after being hospitalised. The difference in experiences throughout the participants' journey battling COVID-19 could have been explained by the improvement in healthcare service provisions and participants' transcendence from victimhood to empowerment. Uncertainty as a source of emotional distress can create opportunities for personal growth in crisis. ${ }^{29}$ In the present study, the experience of living with uncertainty enabled participants to show appreciation for their lives and those of others. In particular, most of the participants expressed deep gratitude to healthcare workers during hospitalisation. This finding differs from Ebola survivors' experiences of abandonment by healthcare workers and healthcare facilities in West Africa. ${ }^{14}$ The healthcare system infrastructure and personnel knowledge and skills in different countries could have led to the difference in findings. In addition, our findings highlighted the importance of early government efforts and commitment to protect people's lives in catastrophic events. The positive feelings that participants held towards healthcare workers during hospitalisation need to be situated and understood within the Chinese social and cultural contexts. Workplace violence against healthcare workers in China has been well documented. $^{3031}$ In Chinese culture, healthcare workers are primarily charged with the responsibilities to treat the disease rather than the patient. Owing to the strict visiting restrictions imposed during the outbreak, family members and caregivers were not allowed to accompany the patients, and nurses took the major responsibility for basic care provision. ${ }^{7}$ While staying on the isolation ward, patients in the present study witnessed healthcare workers' challenging working environments and their dedication to save the lives of others. Our finding strongly supports the positive effect of trusting and understanding relationships between patients and healthcare workers on combating public health crisis. Learning from the participants' experiences, nurses can develop a better understanding of the meaning of presence with and for their patients, the fundamental roles of nurses and the ability of nurses to make significant changes on the lived world of patients. ${ }^{29}$

The dual role of the members of the research team as researchers and caregivers during this inquiry is worth mentioning. Being nurses afforded us upmost closeness to the patients and provided us with the opportunity to share the knowledge discovery process with those under our direct care. Although the research team endeavoured to separate out our clinical and research roles by assigning nurses outside of the research team to recruit participants and conduct interviews, patients might have felt more connected to nursing research activities and answered with more emphasis on nursing care than usual because of our dual role positions. To ensure the voluntary nature of participation and protecting patient well-being, we adopted the ethical practice of 'responsible advocacy' by seeking opinions from healthcare providers to determine an individual's appropriateness of participation. ${ }^{32}$ Beyond the formal ethical guidelines, we were guided in ethical practice through our extensive professional nursing experience.

The limitations of the study must be acknowledged. First, our sample population consisted of patients with mild to moderate clinical symptoms, which limits the transferability of our findings to the COVID-19 patient population at large. The descriptive phenomenological design determined the sample eligibility criteria. However, we recognise the need for further research that includes patients with different severities of the illness. Second, although we made every attempt throughout the study to bracket presuppositions, this alone might not have ensured validity of the findings. One presupposition that needed suspending was based on the fact that members of the research team also provided direct care to the participants. We noticed that participants often perceived us as clinical nurses and automatically kept thanking nurses during the interview. Therefore, we had to constantly remind the participants that we were there to learn from their experiences and what the experiences meant to them. Our reflexive notes also revealed that studying the experience of patients with COVID-19 during the crisis was an emotionally charged project. Nevertheless, our intention to reveal the experiences of those under our care made us determined to brave through the challenges.

\section{Conclusion}

The sudden emergence of COVID-19 has created devastating consequences around the world. In this study, we took a unique opportunity to explore the essence of the experiences of hospitalised patients with COVID-19 following family cluster transmission of the infection in Wuhan, the original epicentre of the pandemic. We were able to conduct the inquiry amidst the infectious outbreak because we were working alongside this patient population as their direct caregivers. Our findings intend to inform the development of specific intervention programmes to prevent survivors from experiencing long-term physical and psychological consequences of the outbreak. Furthermore, this timely investigation of a new phenomenon can generate evidence-based information that might influence the development of guidelines on care provision for patients with COVID-19 in other countries whose outbreak occurred after China.

Acknowledgements The authors acknowledge Tongji Hospital's accommodation of our research requests amidst the COVID-19 outbreak. We would like to thank Chunlin Li, Xiao Wang and Juan Li for their assistance in participant recruitment, data collection and data transcribing. Most importantly, the authors' special thanks go to the patients who participated in this study. Our special thanks also go to the editor and reviewers for their critical comments on an earlier version of the manuscript. 
Contributors WL and JL contributed equally and share the first authorship. JL and WL contributed equally and share the corresponding authorship. JL and WL designed the study. WL and $\mathrm{JL}$ contributed intellectual content during the drafting and approved the final version of the manuscript. CLL, XW and JL provided assistance in participant recruitment, data collection and data transcribing.

Funding This work was supported by National Natural Science Foundation of China (grant number 71904209 to JL) and Adelphi University Faculty Research Release assigned to WL in Spring 2020; grant number not available.

Competing interests None declared.

Patient and public involvement Patients and/or the public were involved in the design, or conduct, or reporting, or dissemination plans of this research. Refer to the Methods section for further details.

Patient consent for publication Not required.

Ethics approval Approval to conduct this study was given by the Institutional Review Board from Tongji Hospital where the study was conducted (stamped and approved) and The Third Xiangya Hospital of Central South University where the researchers were deployed from (no: 2020-S333).

Provenance and peer review Not commissioned; externally peer reviewed.

Data availability statement Data are available on reasonable request. Data used in this study are available from the corresponding authors $\mathrm{JL}$ and $\mathrm{WL}$ upon reasonable request. All personal identifiers found in the data will be removed prior to sharing.

Open access This is an open access article distributed in accordance with the Creative Commons Attribution Non Commercial (CC BY-NC 4.0) license, which permits others to distribute, remix, adapt, build upon this work non-commercially, and license their derivative works on different terms, provided the original work is properly cited, appropriate credit is given, any changes made indicated, and the use is non-commercial. See: http://creativecommons.org/licenses/by-nc/4.0/.

ORCID iD

Wei Liu http://orcid.org/0000-0002-6990-1865

\section{REFERENCES}

1 Wu Z, McGoogan JM. Characteristics of and important lessons from the Coronavirus Disease 2019 (COVID-19) outbreak in China: Summary of a report of 72314 cases from the Chinese Center for Disease Control and Prevention. JAMA 2020;323:1239-42.

2 Wang C, Horby PW, Hayden FG, et al. A novel coronavirus outbreak of global health concern. Lancet 2020;395:470-3.

3 Cucinotta D, Vanelli M. WHO declares COVID-19 a pandemic. Acta Biomed 2020;91:157-60.

4 Chan JF-W, Yuan S, Kok K-H, et al. A familial cluster of pneumonia associated with the 2019 novel coronavirus indicating personto-person transmission: a study of a family cluster. Lancet 2020;395:514-23.

5 Song R, Han B, Song M, et al. Clinical and epidemiological features of COVID-19 family clusters in Beijing, China. J Infect 2020;81:e26-30.

6 Sadang JM. The lived experience of Filipino nurses' work in COVID-19 quarantine facilities: a descriptive phenomenological study. Pacific Rim Int J Nurs Res 2021;25:154-64.

7 Liu Q, Luo D, Haase JE, et al. The experiences of health-care providers during the COVID-19 crisis in China: a qualitative study. Lancet Glob Health 2020;8:e790-8.

8 Sahoo S, Mehra A, Suri V, et al. Lived experiences of the corona survivors (patients admitted in COVID wards): a narrative real-life documented summaries of internalized guilt, shame, stigma, anger Asian J Psychiatr 2020;53:102187-9.

9 Shaban RZ, Nahidi S, Sotomayor-Castillo C, et al. SARS-CoV-2 infection and COVID-19: the lived experience and perceptions of patients in isolation and care in an Australian healthcare setting. Am $J$ Infect Control 2020;48:1445-50.
10 Mansoor T, Mansoor S, Ub Z. 'Surviving COVID-19': illness narratives of patients and family members in Pakistan. Ann King Edward Med Univer 2020;26:157-64.

11 Moradi Y, Mollazadeh F, Karimi P, et al. Psychological disturbances of survivors throughout COVID-19 crisis: a qualitative study. BMC Psychiatry 2020;20:1-8.

12 Ladds E, Rushforth A, Wieringa S, et al. Persistent symptoms after Covid-19: qualitative study of 114 "long Covid" patients and draft quality principles for services. BMC Health Serv Res 2020;20:1144-57.

13 Webber-Ritchey KJ, Simonovich SD, Spurlark RS. COVID-19: qualitative research with vulnerable populations. Nurs Sci $Q$ 2021;34:13-19.

14 Schwerdtle PM, De Clerck V, Plummer V. Experiences of Ebola survivors: causes of distress and sources of resilience. Prehosp Disaster Med 2017;32:234-9.

15 Dowling M. From Husserl to van Manen. A review of different phenomenological approaches. Int J Nurs Stud 2007;44:131-42.

16 Karimi Z, Fereidouni Z, Behnammoghadam M, et al. The lived experience of nurses caring for patients with COVID-19 in Iran: a phenomenological study. Risk Manag Healthc Policy 2020;13:1271-8.

17 Lopez KA, Willis DG. Descriptive versus interpretive phenomenology: their contributions to nursing knowledge. Qual Health Res 2004;14:726-35.

18 Wojnar DM, Swanson KM. Phenomenology: an exploration. J Holist Nurs 2007;25:172-80.

19 Polit DF, Beck CT. Nursing research: generating and assessing evidence for nursing practice. Philadelphia: Wolters Kluwer Health, 2017.

20 Colaizzi PF. Psychological research as the phenomenologist views it. In: Valle RS, King M, eds. Existential-phenomenological alternatives for psychology. New York, NY: Oxford University Press, 1978: 48-71.

21 Tong A, Sainsbury P, Craig J. Consolidated criteria for reporting qualitative research (COREQ): a 32-item checklist for interviews and focus groups. Int J Qual Health Care 2007;19:349-57.

22 Lincoln YS, Guba EG. Naturalistic inquiry. Newbury Park; London; New Delhi: Sage Publications, 1985: 289-331.

23 Quek KM-T, Chen H-M. Family therapy in Chinese culture and context: lessons from supervising therapists-in-training in China. Contemp Fam Ther 2017;39:12-20.

24 National Health Commission of the People's Republic of China. Discharged asymptomatic don't transmit virus, national health body says, 2020. Available: http://en.nhc.gov.cn/2020-06/19/c 80837.htm [Accessed 23 Jul 2020]

25 Rabelo I, Lee V, Fallah MP, et al. Psychological distress among Ebola survivors discharged from an Ebola treatment unit in Monrovia, Liberia - A qualitative study. Front Public Health 2016;4:142-8.

26 National Health Commission of the People's Republic of China. China sends 25,633 medics to battle novel coronavirus in Hubei, 2020. Available: http://en.nhc.gov.cn/2020-02/16/c_76604.htm [Accessed 23 Jul 2020].

27 Xiang Y-T, Yang Y, Li W, et al. Timely mental health care for the 2019 novel coronavirus outbreak is urgently needed. Lancet Psychiatry 2020;7:228-9.

28 Gardner PJ, Moallef P. Psychological impact on SARS survivors: critical review of the English language literature. Can J Psychol 2015;56:123-35.

29 Chung BPM, Wong TKS, Suen ESB, et al. Sars: caring for patients in Hong Kong. J Clin Nurs 2005;14:510-7.

30 Cai R, Tang J, Deng C, et al. Violence against health care workers in China, 2013-2016: evidence from the National judgment documents. Hum Resour Health 2019;17:103-16.

31 Du Y, Wang W, Washburn DJ, et al. Violence against healthcare workers and other serious responses to medical disputes in China: surveys of patients at 12 public hospitals. BMC Health Serv Res 2020;20:253-62.

32 Ulrich CM, Wallen GR, Grady C. Research vulnerability and patient advocacy: balance-seeking perspectives for the clinical nurse scientist? Nurs Res 2002;51:71-2. 